\title{
Psychological research in fibromyalgia: The search for explanatory phenomena
}

\author{
Michael D Boissevain PhD
}

\section{Boissevain.}

Psychological research in fibromyalgia: The search for explanatory phenomena.

Pain Res Manage 1996;1(1):51-57.

This article reviews four areas of psychological research in fibromyalgia. First, the literature on depression in fibromyalgia shows that although the preponderance of studies demonstrate that fibromyalgia patients are more depressed than comparable medical patients, rigorous disconfirming evidence exists. Thus the role of depression in fibromyalgia remains unclear because consistent levels of depression are not found either between samples or between subjects. Second, the role of pain perception in fibromyalgia shows that fibromyalgia patients are consistently more responsive to aversive stimulation than other subjects with chronic pain. This pattern of hyper-responsiveness appears to be generalized, rather than localized to tender points. These results are discussed in terms of potential central nervous system mechanisms. Third, original research in clinical cognitive psychology is presented that shows that fibromyalgia patients do not appear to demonstrate cognitive biases which are distinct from myofascial pain. Although extremely preliminary, these results argue against a unique psychological explanation for fibromyalgia symptoms. Fourth, neuropsychological research demonstrates a pattern of generalized inhibition of information processing that emulates that observed in depressive illness, except that fibromyalgia patients tend not to show the compromise in right hemisphere processing seen in depression. This suggests that cognitive abnormalities in fibromyalgia may be distinct from those seen in depression. The psychological research reviewed suggests that central nervous system anomalies may be implicated in the syndrome of fibromyalgia, but the evidence for motivated or affective psychological involvement in the syndrome remains equivocal. Suggestions are provided for future research.

Key Words: Depression, Fibromyalgia, Pain perception, Psychology

\section{Recherche psychologique en matière de fibromyalgie : Comment pouvons-nous expliquer ce phénomème ?}

RÉSUMÉ : Cet article examine quatre domaines de la recherche psychologique en matière de fibromyalgie. Premièrement, la littérature sur la dépression dans la fibromyalgie met en évidence que bien qu'une prépondérance d'études démontrent que les patients atteints de fibromyalgie sont plus déprimés que ceux atteints de troubles médicaux similaires, il existe également des preuves rigoureuses démentant ces résultats. Par conséquent, le rôle de la dépression dans la fibromyalgie reste inexpliqué parce que l'on n'a pas trouvé des niveaux de dépression logiques soit entre les échantillons ou entre les sujets. Deuxièmement, le rôle de la perception de la douleur dans la fibromyalgie indique que les patients atteints de fibromyalgie sont sans exception plus réceptifs aux stimulations nocives que d'autres sujets accusant une douleur chronique. Cette caractéristique d'hyperréactivité semble être généralisée plutôt que localisée aux points douloureux. Ces résultats sont discutés en terme de mécanismes potentiels du système nerveux central. Troisièmement, les nouveautés en recherche en matière de psychologie clinique cognitive sont présentées et démontrent que les patients atteints de fibromyalgie ne semblent pas accuser des biais cognitifs qui sont distincts de la douleur myofasciale. Bien que très préliminaires, ces résultats témoignent contre une explication psychologique unique pour les symptômes de fibromyalgie. Quatrièmement, la recherche neuropsychologique démontre un schéma d'inhibition généralisée du traitement de l'information qui ressemble à celui observé dans la maladie dépressive, excepté que les patients souffrant de fibromyalgie ont tendance à ne pas présenter le compromis dans le fonctionnement de l'hémisphère droit observé dans la dépression. Ceci laisse croire que les anormalités cognitives observées dans la fibromyalgie pourraient être distinctes de celles observées dans la dépression. La recherche psychologique passée en revue laisse croire que des anomalies du système nerveux central pourraient être impliquées dans le syndrome de la fibromyalgie, mais les preuves en faveur d'une implication psychologique affective ou motivée dans le syndrome restent équivoques. Des suggestions sont fournies pour la recherche future. 
B cause its etiology is unknown, there has been much debate as to whether fibromyalgia is best understood as a medical condition associated with psychological dysfunction or as a psychologically based syndrome associated with 'soft' physiological signs and symptoms. Historically, attempts have been made to define fibromyalgia as a psychogenic pain disorder, wherein the pain symptoms are direct expressions of repressed psychological distress (1). Others have suggested that fibromyalgia may be associated with specific personality characteristics (2) or that fibromyalgia represents a somatic expression of affective disturbance $(3,4)$.

With the publication of the American College of Rheumatology research diagnostic criteria in 1990 (5), and the promise of a reliable and replicable diagnostic schema, the term 'fibromyalgia' appeared to take on some medical and scientific respectability. However, in the intervening years no clear etiological pathway has yet been delineated and no specific physiological markers of the syndrome have been found (6). Thus, the debate persists about the potential etiological role of psychological factors in fibromyalgia. This is exemplified by the inclusion of a chapter on fibromyalgia and myofascial pain in a recent edition of Neurologic Clinics that focused on malingering and conversion reactions (7). In this publication Bohr states that "Despite the fact that FS [fibromyalgia syndrome] patients tend to minimize or deny psychological symptoms, the evidence is overwhelming that the burden of psychiatric disease is higher in comparison to controls".

The extant psychological research was reviewed by Boissevain and McCain in 1991 (8). The conclusions drawn at that time included the following. First, there was no evidence for the existence of distinctive or durable personality characteristics associated with fibromyalgia. Second, when methodologies were employed that simultaneously assessed a number of different indices of psychopathology, subjects diagnosed with fibromyalgia consistently demonstrated higher levels of disturbance than either chronic pain or nonpain controls. However, it was noted that multidimensional psychometric instruments sometimes include items that load on physical symptomatology, thus creating potential interpretational difficulties when applied to a population whose diagnostic symptoms are confounded with putative symptoms of hypochondriasis. Third, after reviewing research on the role of specific affective disturbances in fibromyalgia, including psychometric studies, diagnostic interview studies, and research on biochemical correlates of depression in fibromyalgia syndrome, it was concluded that although some research supported the notion that fibromyalgia is distinguishable from other chronic pain syndromes on the basis of depressive symptomatology, overall the evidence was equivocal. Fourth, it was noted that the most robust difference between fibromyalgia and other painful conditions was in level of reported pain. Across most studies it was found that fibromyalgia patients consistently described their condition as more painful than other chronic pain conditions.

The present paper represents an attempt to update the psychological research in fibromyalgia that has emerged since 1991. As such, it may be seen as a contribution to the debate concerning the potential explanatory roles of psychological factors in the phenomenology of this puzzling syndrome. The literature will be re- viewed selectively, rather than exhaustively, in an effort to focus on areas of research that appear to have the most historical and/or theoretical relevance to this debate. The areas to be reviewed include research on affective disturbance/psychopathology, pain perception, cognitive biases in processing of pain-relevant verbal information and neuropsychology.

\section{FIBROMYALGIA AND DEPRESSION}

Boissevain and McCain (8) suggested that fibromyalgia appears to be consistently associated with higher levels of psychopathology, perhaps through the mechanism of somatization or excessive somatic hypervigilance. However, the picture regarding fibromyalgia and depression was less clear, with inconsistent support found for a relationship between fibromyalgia and the specific syndrome of depression. Since that time the evidence linking depression and fibromyalgia has remained similarly equivocal.

In 1991, Ahles et al (9) conducted structured psychiatric interviews with 35 fibromyalgia patients, 33 rheumatoid arthritis patients and 31 healthy controls. The interviews were conducted in a blinded fashion. Analyses of the interviews revealed no significant differences among the groups on lifetime history of any psychiatric disorder, major depression, somatization disorder or anxiety disorders. Of particular interest for the purpose of the present paper is the finding that $34.3 \%$ of fibromyalgia patients, $39.4 \%$ of rheumatoid arthritis patients and $25.8 \%$ of controls reported a lifetime history of depressive symptomatology. Further, it was found that fibromyalgia patients did not report a higher frequency of vegetative signs of depression than the other two groups.

However, dissenting data have also accumulated since 1991. For example, Hawley and Wolfe (10) compared 6153 rheumatology-practice patients, divided into seven different diagnostic groups, on the depression subscale of the Arthritis Impact Measurement Scale (AIMS). They found that the fibromyalgia group had statistically higher levels of depressive symptomatology than any other group. Further, depending on the cut-off score, between 29.3 and $48.6 \%$ of fibromyalgia patients could be categorized as depressed, again statistically higher than results in any other group. A similar result was observed by Burkhardt et al (11), who found that fibromyalgia patients were significantly more depressed than patients with rheumatoid arthritis $(\mathrm{n}=41$ per group) on the AIMS depression subscale. Similar patterns of results were observed in other studies. Sletvold and colleagues (12) found that fibromyalgia patients were significantly more depressed than normal controls (but significantly less depressed than subjects with major depression) on the Beck Depression Inventory (BDI). On the other hand, Kaplan et al (13) found that fibromyalgia patients obtained a score on the Minnesota Multiphasic Personality Inventory (MMPI) D-scale that was intermediate to, but not significantly different from, a Lyme disease group and a depressed group.

These results suggest that the extent of depression in fibromyalgia is at least partially dependent on measurement methodology. Perhaps the most rigorous study was conducted by Ahles et al (9), who did not find significant differences using a blinded, structured psychiatric interview. This may be compared with re- 
sults obtained by Hawley and Wolfe (10), who reported that positive identification of depression depends on the cut-off score employed. Burkhardt et al (14) have underscored the importance of measurement methodology by comparing four different methods of assessing depression in fibromyalgia patients. They found that a computerized version of the Diagnostic Interview Schedule was the most conservative, estimating the prevalence of depression in a group of 100 fibromyalgia patients at $22 \%$. The most lenient was the BDI, which estimated the prevalence at $55 \%$.

Interestingly, when the BDI was modified by excluding items that directly assess physical symptoms of depression (ie, effort required to accomplish things, sleep disturbance and fatigue), only $29 \%$ of the patients were classified as depressed, thus underscoring the importance of partialling out the effect of vegetative symptoms when assessing depression in medical patients. These results also suggest that caution should be employed when interpreting results obtained in a multicentre trial conducted by Ercolani et al (15). Although this group classified $49 \%$ of their sample of 327 fibromyalgia patients as depressed, they employed a cutoff score of 23 on the Centre for Epidemiologic Studies Depression Scale.

There does not appear to be any clear resolution of questions regarding the potential etiological role of depression in fibromyalgia. It is evident that depression is a problem for many individuals with a diagnosis of fibromyalgia, but two other confounding observations are also evident. First, a significant proportion of fibromyalgia patients (perhaps most fibromyalgia patients) do not become depressed. This suggests that depression is not an invariant component in the expression of the syndrome. Second, average levels of depression in fibromyalgia patients consistently appear to be lower than average levels of depression observed in clinically depressed psychiatric comparison groups. This may suggest that the depression observed in fibromyalgia patients may be qualitatively, as well as quantitatively, distinct from the clinical entity of depression. It is possible, as suggested by Hudson and Pope (16), that fibromyalgia may be affected by an underlying pathophysiological abnormality in common with other affectively based syndromes. However, it may also be possible that fibromyalgia is not a monolithic entity, but that it subsumes a broad spectrum of pain disorders, some of which may be driven by a significant affective/psychological component and some of which may be more heavily mediated by organic factors. To date, however, our diagnostic tools have not permitted a finer grained analysis of this population.

\section{PAIN PERCEPTION}

Since 1991, two studies have been conducted in Gary Rollman's laboratory at the University of Western Ontario that examined the ways in which fibromyalgia patients respond to aversive stimulation compared with both normal controls and other chronic pain patients. These studies may be seen as the latest in a series that was begun in 1987 with the publication of a paper by Scudds et al (17), in which it was shown that fibromyalgia subjects had lower tolerance for dolorimeter-induced pain than either rheumatoid arthritis patients or healthy controls ( $\mathrm{n}=20$ for all groups). Fibromyalgia patients had a lower threshold for dolorimeter-induced pain than normal controls. Similar trends were observed for experi- mental pain induced by constant pressure algometer and cutaneous electrical stimulation. Because the fibromyalgia subjects were assessed as more psychologically disturbed than comparison groups, Scudds et al (17) viewed these results as suggesting that pain perception in fibromyalgia may be influenced by both pain hyper-responsivity and psychological disturbance.

In 1994 Lautenbacher et al (18) compared 26 fibromyalgia patients with 26 matched healthy controls on threshold sensitivity for noxious and nonpainful stimuli on an upper trapezius tender point site and a nontender control point site located on the inner forearm. Painful stimulation was induced by dolorimetry pressure, by heat via a contact thermode and by cutaneous electrical pain. Nonaversive stimulation included warmth, cold and nonpainful electrical stimulation. It was generally found that relative to healthy subjects, fibromyalgia patients exhibited lower thresholds for painful stimulation at both tender and control points, when induced by pressure or heat. For cutaneous electrical stimulation, fibromyalgia patients had lower thresholds only on the tender point. Detection thresholds for cold was lower for fibromyalgia patients than for normals. However, for warmth or nonaversive electrical stimulation, no between-group differences were observed.

Lautenbacher et al (18) also examined correlations between induced pain threshold and self-reported clinical pain, as assessed by the McGill Pain Questionnaire, and the Localized Pain Rating. Only four correlations of an array of 48 were significant, but they were relatively modest in magnitude, thus suggesting very little relationship between pain tolerance and experienced pain. Because fibromyalgia patients were responsive to all aversive stimulation across both sites, Lautenbacher et al argued that these results supported the notion that fibromyalgia patients are generally hypersensitive to noxious stimulation, and that this hyper-responsivity may be due to both central and peripheral factors.

A more recent study has expanded upon the issue of generalized hypervigilance in fibromyalgia by examining threshold and tolerance for dolorimeter-induced pain, and auditory tolerance for aversive noise (19). In this study, fibromyalgia and rheumatoid arthritis patients were compared with healthy controls $(n=20$ per group). Instead of examining tender points, dolorimeter pressure was applied to the antebrachial area of the forearm bilaterally. Results showed that the fibromyalgia group had a significantly lower threshold (mean $1.51 \mathrm{~kg}$ ) than the rheumatoid arthritis group (mean $2.89 \mathrm{~kg}$ ) and the controls (mean $5.50 \mathrm{~kg}$ ). The rheumatoid arthritis group was also found to have a significantly lower threshold than the control group. Analyses of pain tolerance revealed a similar pattern, with all between-group comparisons significant. The fibromyalgia group tolerance was 2.78 $\mathrm{kg}$; for the rheumatoid arthritis group it was $4.59 \mathrm{~kg}$ and for the controls $8.05 \mathrm{~kg}$. In examining the effect of tolerance for aversive noise a similar pattern was observed. The fibromyalgia group had a significantly lower noise tolerance (mean $66.17 \mathrm{~dB}$ ) than the rheumatoid arthritis group (mean $75.98 \mathrm{~dB}$ ) or the control group (mean $100.48 \mathrm{~dB}$ ). The rheumatoid arthritis group also obtained a significantly lower tolerance level than the controls.

Granges and Littlejohn (20) also conducted an interesting study that may assist in shedding some light on the nature of the 
pain modulation response in fibromyalgia. They tested algometry pressure pain thresholds in fibromyalgia patients, patients with regional pain symptoms and nonpain controls ( $n=60$ per group). Thresholds were tested on the 18 tender points specified by the 1990 American College of Rheumatology diagnostic criteria (5), as well as on four control points (thumbnails and mid-deltoids, bilaterally). It was found that the fibromyalgia patients had the lowest pressure-pain thresholds, both on tender and control points, as well as when control and tender point scores were combined. However, few subjects, including the fibromyalgia subjects, demonstrated a threshold over control points that was lower than $4 \mathrm{~kg} / \mathrm{cm}^{2}$, which had been predetermined as the operational definition of 'tender'. The mean thresholds over control points were $4.8 \mathrm{~kg} / \mathrm{cm}^{2}, 6.5 \mathrm{~kg} / \mathrm{cm}^{2}$ and $8.4 \mathrm{~kg} / \mathrm{cm}^{2}$ for the fibromyalgia group, the regional pain group and the control group, respectively. Interestingly, the threshold over tender points was significantly lower than the threshold over control points for all groups: mean $1.7 \mathrm{~kg} / \mathrm{cm}^{2}$ for the fibromyalgia group, mean $2.9 \mathrm{~kg} / \mathrm{cm}^{2}$ for the regional pain group and mean $4.3 \mathrm{~kg} / \mathrm{cm}^{2}$ for the control group. Granges and Littlejohn (20) interpreted these findings as suggesting that fibromyalgia is likely to be a centrally mediated disorder of pain modulation rather than a localized peripheral abnormality. Fibromyalgia thus represents a generalized reduction in pain threshold, although this reduction is most apparent over tender points.

Finally, Arroyo and Cohen (21) tested the effects of cutaneous electrical stimulation on 10 fibromyalgia patients and 10 healthy controls. Stimulation was induced bilaterally at four points on the arm and two points on the neck. These loci were chosen to test dermatomal innervation patterns, rather than to assess tender points. It was found that groups did not differ in threshold, but that tolerance was lower in fibromyalgia subjects. Unexpectedly, it was also found that fibromyalgia patients experienced a distal and proximal spread of sensation following stimulation, and that fibromyalgia patients reported that these sensations persisted from $12 \mathrm{~s}$ to $20 \mathrm{mins}$. Arroyo and Cohen (21) interpreted these results as suggesting that electrocutaneous stimulation may create a state of excitation in afferent neurons. However, the design of their study did not permit a distinction between central and peripheral mechanisms.

Taken together, results from the psychophysics research suggest that chronic pain confers a lowered tolerance for aversive stimulation, not only in the somatosensory modality, but also in the auditory one. In the case of fibromyalgia, however, there consistently appears to be a more extreme hypervigilant response to aversive stimulation than in other chronic pain conditions. The mechanisms for this are not clear. However, Lautenbacher et al (18) suggested that the observed pattern of pain hyperresponsiveness suggests an interaction between central and peripheral factors. Hypothesized central nervous system (CNS) factors include an inability to inhibit the perception of painful stimulation (22), generalized hypervigilance to aversive somatic events (18) and psychological factors that may amplify pain signals (20,23). Arroyo and Cohen (21) echoed these suggestions, and stated that "FS may be an example of altered central nociception, perhaps associated with peripheral nociception, though the relevant mechanisms remain to be identified".

\section{COGNITIVE CLINICAL PSYCHOLOGY}

Cognitive approaches in clinical psychology are predicated on the notion that specific information processing biases are implicated in various affective disturbances. Thus, it has been amply demonstrated, for example, that depressives are more likely than nondepressed subjects to attend to information with depressionrelevant content, to recall personal memories that are congruent with depressed mood and to be biased towards excessive false recognition of depression-relevant stimuli (for a review, see Williams et al [24]). Research is emerging that demonstrates that similar information processing biases may be present in chronic pain.

It should be noted that the term 'information processing' in clinical psychology is conceptually different from the neuropsychological use of the term. In the case of clinical psychology, the 'information' is semantic content that relates to the particular pathological condition under consideration. Thus cognitive clinical psychology is not necessarily concerned with specific neurological or cortical anomalies, but is instead concerned with psychological processes. In neuropsychology, on the other hand, there is less concern with the semantic content of the information', and more concern with whether the anomalous processes are indicative of a specific locus of CNS compromise.

Research has generally shown that individuals suffering from specific affective disturbances are biased in their processing of information that is semantically related to the disturbance in question. For example, depressed individuals are biased towards information that is semantically related to the concept of depression, whereas spider phobics are biased towards information that relates to their specific phobia. These biases have been shown to be robust across a number of different levels of cognitive processing, including attention $(25,26)$, short term recognition memory (27) and long term autobiographical memory (28). Similar phenomena have also begun to be demonstrated in chronic pain, where it has been shown that subjects experiencing pain are predisposed to attending to $(29)$, to remembering $(30,31)$ and to recognizing pain-relevant stimuli (32).

In an unpublished doctoral dissertation, Boissevain (33) examined information processing biases in depressed and nondepressed groups of subjects with heterogeneous chronic pain conditions compared with depressed psychiatric patients and healthy controls. These data were reanalyzed in order to compare a sample of fibromyalgia patients $(n=25)$ with a sample of myofascial pain syndrome patients $(n=24)$ and a sample of nonpain controls $(n=15)$ on tasks that examined potential biases in the processing of pain-congruent and noncongruent verbal stimuli. Processes examined included attention, as assessed by a modified Stroop task, an autobiographical memory task and an incidental recognition memory test. On the Stroop task, negative words, positive words and sensory pain words were presented on separate cards. Ten words from each category were used, with each word repeated eight times on each card, and printed in red, green, orange or blue ink. Words had been standardized and normed in previous research. Subjects were required to name the colour in which the word was printed while trying to ignore the semantic content of the word. The dependent measure was the time taken to name the colours on each of the three cards. In the autobiographi- 
cal memory task, subjects were auditorially cued with words from the same three categories (ie, positive, negative and sensory pain). Subjects were requested to recall a cue-relevant personal memory immediately following presentation of the cue. The dependent measure in this task was the latency to recall the personal memory. In the incidental recognition task, verbal stimuli were presented on a page that contained four columns of 25 words each. Target words were the five words presented in the autobiographical memory task. The word list also contained 10 distracters from each of the categories in the earlier tasks, as well as 55 neutral distracters. Subjects were required to check words that they believed had been presented in the autobiographical memory task. Dependent variables in this task were proportion of target stimuli correctly identified (hits) and proportion of distracters in each target category that were incorrectly identified (false alarms).

Analyses showed that the interaction terms for the Stroop task failed to attain statistical significance, thus suggesting that diagnostic status did not confer any domain-specific attentional bias. On the autobiographical memory task, the interaction term did attain significance, with post hoc analyses showing that the fibromyalgia and myofascial pain groups were similar in showing faster response times for pain-relevant stimuli. These results suggest that the experience of pain, regardless of diagnosis, allows pain-relevant personal memories to be more accessible to retrieval. In analyzing the proportion of correct recognitions (ie, hit rate) on the incidental recognition task, the group/stimulus type interaction term was significant. Post hoc analyses showed that the fibromyalgia group more accurately recognized pain stimuli than positive stimuli; that the hit rate for pain stimuli was higher than for negative stimuli in the myofascial group; and that the hit rate for positive stimuli was higher than that for pain stimuli in controls. These results again suggest that the experience of pain, rather than diagnostic category, may prime recognition memory for pain-relevant stimuli. When analyzing the proportion of incorrect recognitions (ie, false alarms), no significant group-based differences emerged, thus suggesting that chronic pain may not confer a bias in recognition of pain-relevant stimuli.

Although these results must be viewed as extremely preliminary in the examination of cognitive biases in fibromyalgia, the data suggest that in general, the experience of pain may create a predisposition to attending to, remembering and recognizing pain-relevant verbal information. This observation suggests that pain can influence the processing of pain-relevant information, analogous to the way in which affective disturbance can influence the processing of emotion-relevant information. It is notable that no significant differences emerged when comparing fibromyalgia with myofascial subjects. This result suggests that fibromyalgia symptomatology is probably not mediated by higher level psychological processes, such as attention, memory or judgement, to a greater extent than is myofascial pain syndrome. However, it must be acknowledged that myofascial pain may not be the most appropriate comparison group for psychological research on fibromyalgia because both conditions are sometimes understood as having similar levels of psychological involvement in their presentation $(7,34)$.

\section{NEUROPSYCHOLOGICAL RESEARCH}

Two recently published papers have examined the possibility that fibromyalgia sufferers may have cognitive deficits when assessed by neuropsychological assessment instruments. The general thesis presumed by neuropsychological research is that the presence of specific cognitive deficits in fibromyalgia helps to demonstrate that fibromyalgia symptoms may be explicable in terms of physiologically based CNS dysfunction, as opposed to an affective reaction to pain.

In 1992, Kaplan et al (13) compared 20 patients with Lyme disease encephalopathy (a syndrome with a specific, identifiable organic etiology) with 11 patients with fibromyalgia and 11 depressed subjects without psychotic symptoms. Three tests of memory were used: the Wechsler Memory Scale (WMS), the California Verbal Learning Test (CVLT) and the Rey-Osterrieth Complex Figure Test. Subjects were also administered the MMPI and the BDI. Overall, there were few differences among the three groups. Differences that did emerge, however, showed that the Lyme disease patients had more impaired memory than either the fibromyalgia patients or the depressives. Specifically, the Lyme disease patients performed significantly poorer than depressives on the paired-associated learning subtest of the WMS, poorer than the fibromyalgia patients on trial 5 of the CVLT, and poorer than both fibromyalgia subjects and depressives on the $20 \mathrm{~min}$ delayed recall of the CVLT.

Interestingly, despite the poorer cognitive performance on the part of the Lyme subjects, they reported significantly less psychological distress than the other two groups on the MMPI. In fact, the Lyme disease group did not obtain any $\mathrm{T}$ scores in the clinical range $(\mathrm{T}>70)$. Of particular note is the observation that the fibromyalgia group expressed significantly more somatic symptomatology than the Lyme group on the two MMPI scales that assess somatic distress, ie, on Scale 1 (Hysteria) and Scale 2 (Hypochondriasis). Kaplan et al (13) noted that the MMPI profile produced by the Lyme patients was similar to those produced by other medical patients, and showed little tendency towards psychological disability as a consequence of their illness. On the BDI, Lyme patients obtained lower scores (mean 7.30) than fibromyalgia patients (mean 13.66), who in turn obtained lower scores than depressives (mean 16.66), although these differences failed to attain statistical significance. The investigators felt that the objective memory impairments manifested by Lyme patients were likely due to an underlying disease process, whereas the memory complaints made by both fibromyalgia patients and depressives were more subjective than quantifiable and were likely due to affective disturbance.

The second recent neuropsychological study was published by Sletvold et al (12). Groups included fibromyalgia patients $(n=25)$, depressives $(n=22)$ and healthy subjects $(n=18)$. In this study a number of specific cognitive processes were investigated. Psychomotor speed was assessed by the Digit Symbol Test from the Wechsler Adult Intelligence Scale (WAIS). Psychomotor speed and attention were assessed by the Trail Making Test. Working memory was assessed by the Paced Auditory Serial Addition Task. Simple reaction times were also recorded. Finally, right hemisphere function was assessed by the Block Design Task from the WAIS and left hemisphere function was assessed 
by the WAIS Similarities Task. Groups were also given a semistructured psychiatric diagnostic interview and the BDI.

Results showed that all groups differed significantly on depression levels, with the depressed group highest, followed by the fibromyalgia group and the controls. In terms of neuropsychological functioning, the fibromyalgia patients were similar to the depressives; both groups demonstrated a generalized information processing deficit across all tasks relative to healthy controls. Interestingly, when fibromyalgia subjects were separated into a depressed and a nondepressed group, there were no differences between groups on any of the cognitive tasks. This finding was interpreted as suggesting that the generalized information processing dysfunction in fibromyalgia may not have been completely mediated by depression. The only pattern of test responses that distinguished fibromyalgia subjects from depressives was in the WAIS-R Similarities and Block Design Subtests, where the depressives performed more poorly on Block Design than on Similarities, thus suggesting a relative right hemisphere deficit. Fibromyalgia subjects showed no difference across these two tests. Sletvold et al (12) argued that this pattern of results may mean that fibromyalgia and depression are biologically different conditions in terms of CNS activation because fibromyalgia patients were distinguishable from depressives on the basis of tests that assess lateralized brain function.

The results of these two studies suggest that fibromyalgia shares with depression a generalized information processing deficit. However, the memory compromise seen in fibromyalgia may not be unique to this disorder and may, in fact, be less severe than in similar disorders with an identifiable organic basis, such as Lyme encephalopathy. In the two studies cited here, the one feature that distinguishes fibromyalgia from depression is that fibromyalgia appears to be characterized by a generalized deficit on tasks that measure lateralization of brain function, whereas major depressive disorder appears to be characterized by compromised right hemisphere processing. However, it must be acknowledged that observed differences were relatively weak and may not have clinical significance. Although these results suggest that unique, potentially explanatory cognitive abnormalities may not exist in fibromyalgia, similar research with more diverse comparison groups is needed before this question can be answered definitively.

\section{DISCUSSION}

Since its initial recognition as a distinct diagnostic entity, researchers have searched for psychological variables that could explain the etiology and maintenance of fibromyalgia. Initial research was relatively unsophisticated and conceptualized fibromyalgia as a direct expression of psycholopathology, in much the same fashion as conversion disorder. Later research has looked into the potential role of affective disturbance, especially depression. However, as noted by Boissevain and McCain (8), although fibromyalgia is reliably associated with depression and other forms of affective disturbance, the evidence that fibromyalgia is more strongly associated with depression than many other chronic pain syndromes has been inconsistently supportive. Research that has emerged since 1991 appears to be similarly equivocal; the extent of depression observed in fibromyalgia may depend on assessment methodology, sampling characteristics and comparison groups.

Other psychological research on fibromyalgia that has emerged since 1991 appears to be more sophisticated in the variables considered and their potential relationship to fibromyalgia symptomatology. Three recent research directions were considered in the present paper.

In their review, Boissevain and McCain (8) suggested that the most reliable distinction between fibromyalgia and most other chronic pain disorders was in the level of pain expressed by fibromyalgia patients; across different laboratories, cultures and measures, fibromyalgia is consistently described as more painful than other chronic pain conditions. Two of the areas reviewed in the present paper examined recent research that has attempted to delineate potential psychological reasons for the pain phenomenology of fibromyalgia. First, psychophysical methodology has been used to examine perceptual processes in fibromyalgia. This research has shown that fibromyalgia is associated with lower thresholds for painful stimulation than other chronic pain conditions (17), that this threshold difference is robust across different pain induction methods (18), that this difference also extends to tolerance for aversive auditory stimulation (19) and that this difference may represent a generalized hypersensitivity to aversive stimulation (20). Second, a study was reviewed that employed cognitive clinical psychology methodology to examine the potential for information processing that was biased in favour of pain-relevant stimuli (34). This research is predicated on the notion that expressed pain may be the result of cognitive biases, similar to the cognitive biases seen in affective disturbance. Results from this research suggest that fibromyalgia is not distinct from myofascial pain in terms of information processing bias, but that chronic pain in general may be associated with cognitive biases for state-congruent stimuli.

Finally, the neuropsychological research reviewed did not explicitly consider the role of pain per se in its research questions. Some results from this area suggest that memory impairment in fibromyalgia may be more strongly associated with affective disturbance than with an underlying disease process (13). However, later research suggests that fibromyalgia is associated with a generalized information processing deficit that is not due to depression and that does not show the right hemisphere deficit commonly seen in depression (12).

It appears that all these areas of research may represent fruitful lines of inquiry in fibromyalgia. In terms of depression research, it may be useful to attempt further comparisons between depressed and nondepressed fibromyalgia patients. The commonalities and differences between these two groups are not yet clear, and such investigations may inform both etiological understanding and treatment. Fibromyalgia is associated with more pain than other similar conditions, and it is associated with lower thresholds for induced pain. These findings have led McDermid et al (19) to refer to the hypervigilance model of pain perception developed by Chapman (35) that states that chronic pain is associated with heightened pain sensitivity due to aberrant CNS processes. Lautenbacher et al (18) suggested that the experience of fibromyalgia symptoms is likely due to an interaction between central and peripheral mechanisms, an hypothesis echoed by 
Yunus (22). It would be useful if future research attempts to delineate the locus of the CNS disturbance that seems to characterize fibromyalgia. That the present cognitive clinical results were negative suggests that the locus of disturbance may not be in the prefrontal association cortex, where judgement, perception, language and thinking processes are believe to be localized (36). However, the fact that cognitive clinical psychology appears to offer some treatment possibilities (37) suggests that it may be possible to delineate further the active cognitive ingredients in the phenomenology of the syndrome. It is also possible that information processing anomalies in fibromyalgia may become apparent if more diverse comparison groups are employed in such

\section{REFERENCES}

1. Boland EW. Psychogenic rheumatism: the musculoskeletal expression of psychoneurosis. Ann Rheumatol Dis 1947;6:195.

2. Smythe HA. "Fibrositis"" and other diffuse musculoskeletal syndromes. In: Kelly WN, Harris ED, Ruddy S, Sledge CB, eds. Textbook of Rheumatology. Philadelphia: Saunders, 1985:481-9.

3. Blumer D, Heilbrun M. Chronic pain as a variant of depressive disease: the pain-prone disorder. J Nerv Ment Dis 1982;170:381-406.

4. Diamond HS. Psychogenic rheumatism. Clin Rheumatol Pract 1984:2:131-3.

5. Wolfe F, Smythe HA, Yunus MB, et al. The American College of Rheumatology 1990 criteria for the classification of fibromyalgia. Arthritis Rheum 1990;33:160-72.

6. Henriksson KG. Pathogenesis of fibromyalgia. J Musculosk Pain 1993;1:3-15.

7. Bohr TW. Fibromyalgia syndrome and myofascial pain syndrome: do they exist? Neurologic Clin 1995;13:365-84.

8. Boissevain MD, McCain GA. Toward an integrated understanding of fibromyalgia syndrome. II: psychological and phenomenological aspects. Pain 1991;45:239-48.

9. Ahles TA, Khan SA, Yunus MB, Spiegel DA, Masi AT. Psychiatric status of patients with primary fibromyalgia, patients with rheumatoid arthritis, and subjects without pain: a blind comparison of DSM-III diagnoses, Am J Psychiatry 1991;148:1721-6.

10. Hawley DJ, Wolfe F. Depression is not more common in rheumatoid arthritis: a 10-year longitudinal study of 6,153 patients with rheumatic disease. J Rheumatol 1993;20:2025-31.

11. Burkhardt CS, Clark SR, Bennett RM. A comparison of pain perceptions in women with fibromyalgia and rheumatoid arthritis: relationship to depression and pain extent. Arth Care Res 1992;5:216-22.

12. Sletvold H, Stiles TC, Landro NI. Information processing in primary fibromyalgia, major depression and healthy controls. J Rheumatol 1995;22:137-42.

13. Kaplan RF, Meadows ME, Vincent LC, Logigian EL, Steere AC. Memory impairment and depression in patients with Lyme disease encephalopathy. Neurology 1992;42:1263-7.

14. Burkhardt CS, O'Reilly CA, Wiens AN, et al. Assessing depression in fibromyalgia patients. Arth Care Res 1994;7:35-9.

15. Ercolani M, Trombini G, Chattat R, et al. Fibromyalgia syndrome: depression and abnormal illness behavior. Psychother Psychosom 1994; $161: 178-86$.

16. Hudson JI, Pope HG. Fibromyalgia and psychopathology: is fibromyalgia a form of 'affective spectrum disorder'? J Rheumatol 1989;16(Suppl 19):15-22.

17. Scudds RA, Rollman GB, Harth M, McCain GA. Pain perception and personality measures as discriminators in the classification of fibrositis. J Rheumatol 1987;14:563-9.

18. Lautenbacher S, Rollman GB, McCain GA. Multi-method assessment research. Similarly, the extant neuropsychological research also suggests that fibromyalgia is not associated with a lateralized cognitive deficit. Thus there may be other specific CNS structures that are more likely to be associated with the observed hypervigilance for aversive stimulation.

Finally, however, the accumulated evidence suggests that fibromyalgia cannot be wholly explained by psychological factors. It is clear that psychological factors play an important role in the maintenance and phenomenology of the syndrome, but it is not clear whether they are involved in its etiology. It appears that further answers to the question of the psychology of fibromyalgia must await more refined diagnostic criteria and prospective studies.

of experimental and clinical pain in patients with fibromyalgia. Pain. 1994;59:45-53.

19. McDermid AJ, Rollman GB, McCain GA. Generalised hypervigilance in fibromyalgia: Evidence of perceptual amplification. Pain. (In press)

20. Granges G, Littlejohn G. Pressure pain threshold in pain-free subjects, in patients with chronic regional pain syndromes and in patients with fibromyalgia syndrome. Arthritis Rheum 1993;36:642-6.

21. Arroyo JF, Cohen ML. Abnormal responses to electrocutaneous stimulation in fibromyalgia. J Rheumatol 1993;20:1925-31.

22. Yunus MB. Towards a model of pathophysiology of fibromyalgia: aberrant central pain mechanisms with peripheral modulation. J Rheumatol 1992;19:846-50.

23. Epidemiological research on the etiological factors behind the dysfunctional diseases of the motor apparatus: the stomatogenate system as an example. In: Muller W, ed. Generalisierte Tendomyopathie (Fibromyalgie). Darmstadt: Steinkopff Verlag, 1991:221-9.

24. Williams JMG, Watts FN, MacLeod C, Matthews A. Cognitive Psychology and the Emotional Disorders. Chichester: Wiley, 1988.

25. Matthews A, MacLeod C. Selective processing of threat cues in anxiety states. Behav Res Ther 1985;23:283-302.

26. Williams JMG, Nulty DD. Construct accessibility, depression, and the emotional Stroop task: transient mood or stable structure? Pers Ind Diff 1986;7:485-91.

27. Dunbar GC, Lishman WA. Depression, recognition memory, and hedonic tone: a signal-detection analysis. Br J Psychiatry 1985;144:376-82.

28. Williams JMG, Dritschel BH. Emotional disturbance and the specificity of autobiographical memory. Cogn Emot 1988;2:221-34.

29. Pearce J, Morley S. An experimental investigation of the construct validity of the McGill Pain Questionnaire. Pain 1989;43:187-93.

30. Erskine A, Morley S, Pearce J. Memory for pain: a review. Pain 1990;43:255-65.

31. Eich E, Rachman S, Lopatka C. Affect, pain, and autobiographical memory. J Abnormal Psychol 1990;99:174-8.

32. Edwards L, Pearce J, Collet B, Pugh R. Selective memory for sensory and affective information in chronic pain and depression. Br J Clin Psychol 1992;31:239-48.

33. Boissevain MD. Information Processing in Chronic Pain: The Role of Depression. London: University of Western Ontario, 1994.

34. McCain GA, Scudds RA. The concept of primary fibromyalgia (fibrositis): clinical value, relation and significance to other musculoskeletal pain syndromes. Pain 1988;33:273-87.

35. Chapman CR. Pain: The perception of noxious events. In: Sternbach RA, ed. The Psychology of Pain. New York: Raven, 1978:169-202.

36. Lezak MD. Neuropsychological Assessment, 3rd edn. New York: Oxford, 1995.

37. White KP, Nielson WR. Cognitive behavioral treatment of fibromyalgia syndrome: a follow-up assessment. J Rheumatol $1995 ; 22: 717-21$ 


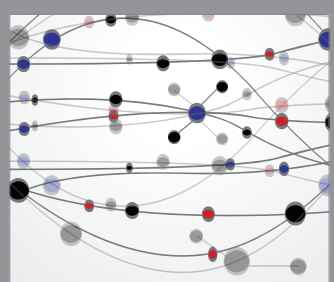

The Scientific World Journal
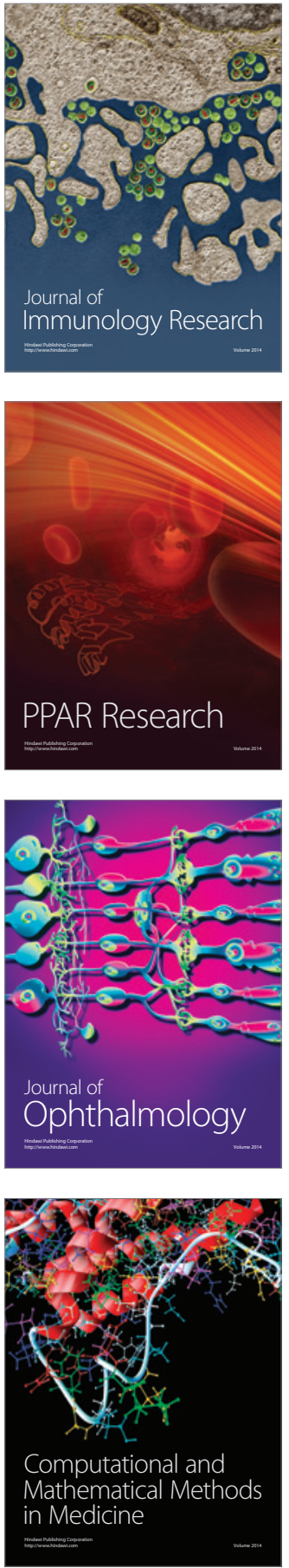

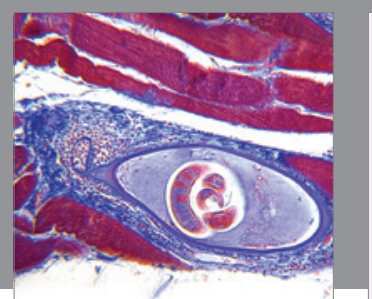

Gastroenterology Research and Practice

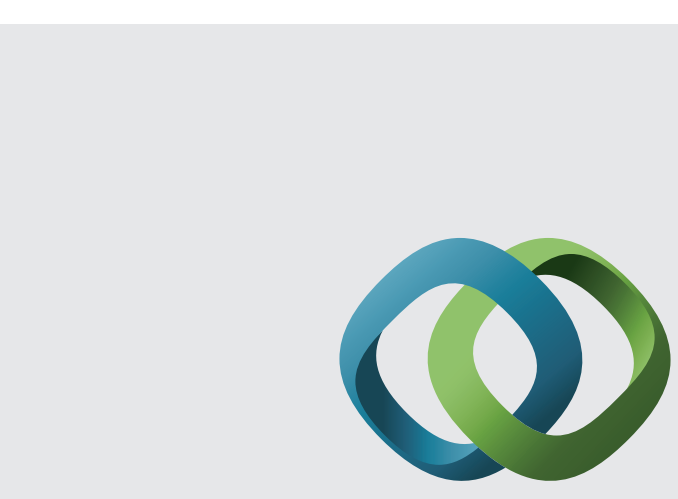

\section{Hindawi}

Submit your manuscripts at

http://www.hindawi.com
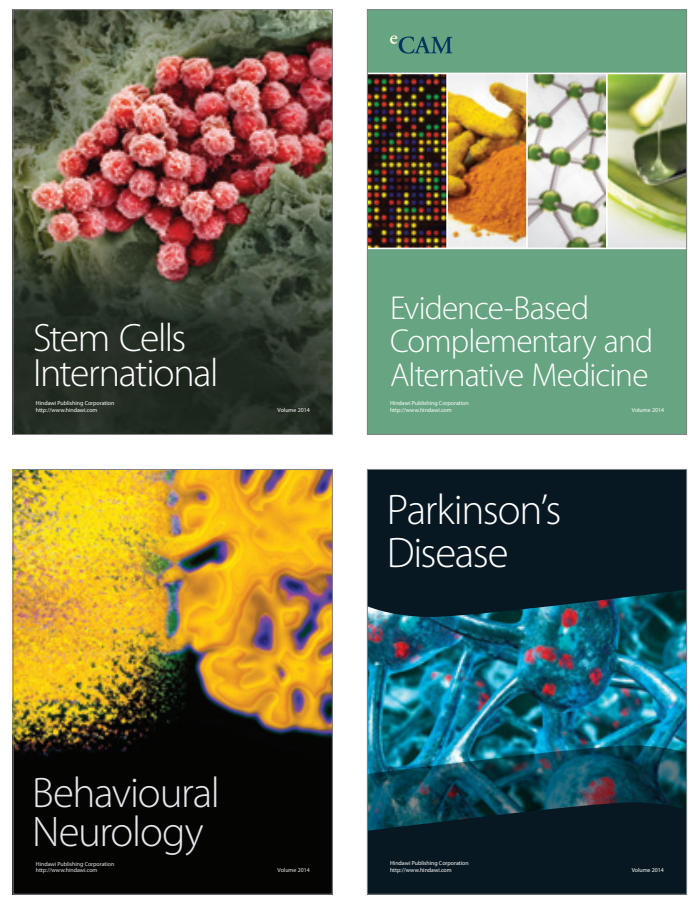
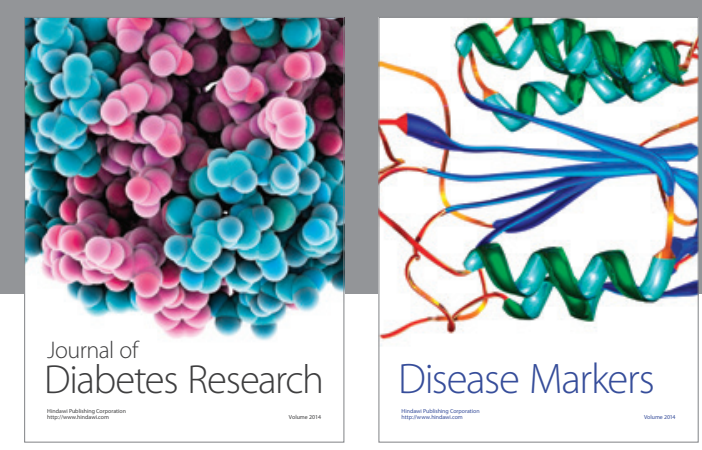

Disease Markers
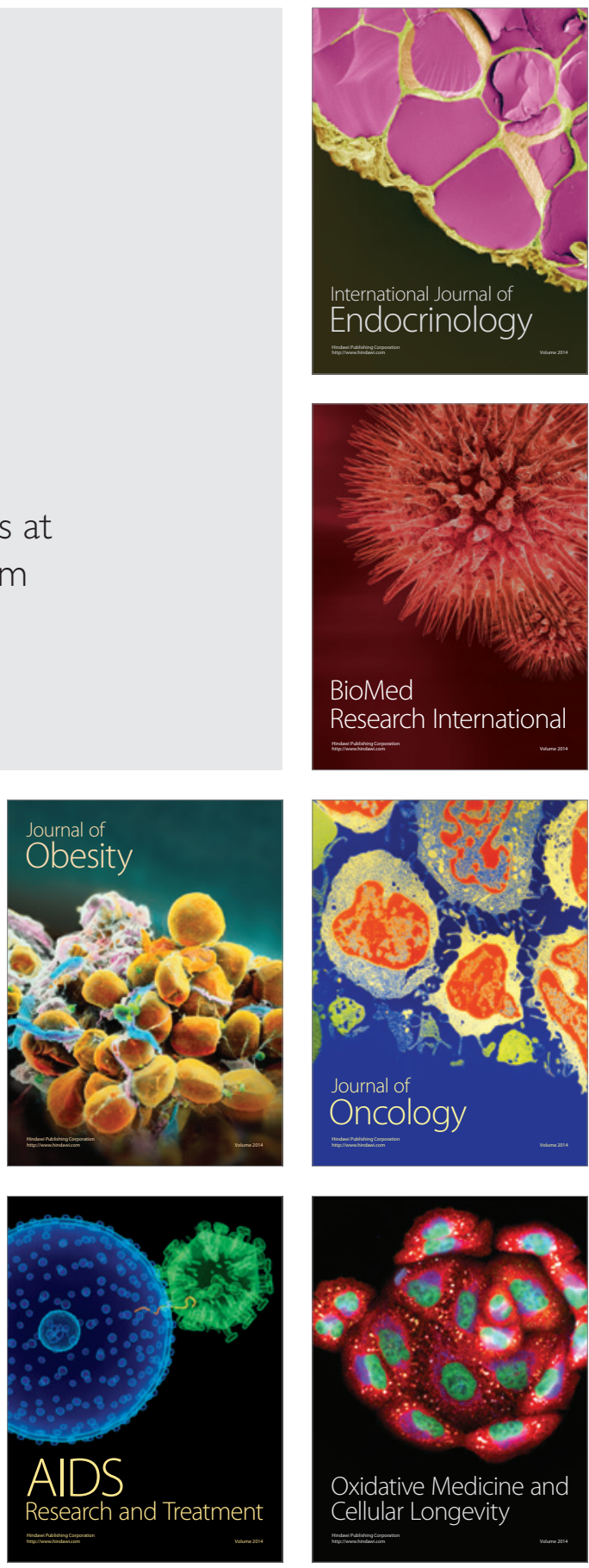